\title{
Determining mode-I cohesive parameters for nugget fracture in ultrasonic spot welds
}

\author{
BIN ZHOU ${ }^{1}$, M.D. THOULESS ${ }^{1,2}$ and S.M. WARD ${ }^{3}$ \\ ${ }^{1}$ Department of Mechanical Engineering, University of Michigan, Ann Arbor, MI 48109, USA \\ ${ }^{2}$ Department of Material Science and Engineering, University of Michigan, Ann Arbor, MI 48109, USA \\ ${ }^{3}$ Scientific Research Laboratory, Ford Motor Company Dearborn, MI 48124, USA
}

Received 15 August 2005; accepted in revised form 20 December 2005

\begin{abstract}
Ultrasonic spot welds have been used as a model system to investigate how to determine the mode-I cohesive parameters associated with interfacial fracture of a spot weld. Numerical analyses indicated that, while multiple combinations of the two cohesive parameters (characteristic strength, $\hat{\sigma}$, and toughness, $\Gamma_{I}$ ) could result in virtually indistinguishable behaviors for individual geometries, only a single pair of parameters can provide a unique set of behaviors for different test geometries. This provides the basis for determining the cohesive parameters by comparing numerical predictions to experimental observations. In particular, a direct uniaxial tensile test was found to be particularly useful for measuring the characteristic strength of an ultrasonic weld. With the characteristic strength known, the toughness of the weld was determined by fitting numerical predictions to experimental observations of the load-displacement curves obtained from T-peel specimens bonded with the ultrasonic weld. These two parameters were then used without modification to predict the performance of welded U-peel specimens. The numerical predictions for this third configuration were in excellent agreement with the experimental results, verifying that it may be possible to use cohesive-zone parameters to predict the behavior of different geometries of spot welds formed under nominally similar conditions.
\end{abstract}

Key words: Cohesive zone, fracture, plastic deformation, spot weld, ultrasonic.

\section{Introduction}

Aluminum alloys can provide superior performance to steels for automotive applications, in terms of higher strength-to-weight ratios, lighter weights and higher resistance to corrosion (Hetrick et al., 2005). Unfortunately, aluminum alloys are more difficult to join using conventional welding techniques. Ultrasonic spot welding (USW) has recently been recognized as a promising technology for welding aluminum sheet in automotive structures. Ultrasonic lateral vibrations $(\geqslant 20 \mathrm{kHz})$ of a welding tip, combined with a static clamping pressure, can lead to a solid-state metallurgical bond being formed across the interface between two components, without any melting occurring (Harthoorn, 1978). The crucial process parameters that appear to contribute significantly to the weld strength are the welding energy and time, the vibration frequency, the clamping pressure, and the size of the welding tip (Jones and Meyer, 1958; Hazlett and Ambekar, 1970; Harthoorn, 1978; Matsuoka, 1995; Tsujino et al., 2002). It also appears that the strength of the weld is directly related to the bonded area. In the present work, all the welding parameters were kept constant to provide a model welded interface. 


\section{B. Zhou et al.}

Most previous studies on the strength of spot welds (Satoh et al., 1991; Lee et al. 1998; Chang et al., 1999, Deng et al., 2000; Lin et al., 2001; Wung, 2001; Wung et al., 2001) have employed strength-based approaches, where failure criteria are formulated in terms of the local loads acting on the welds. However, it is known that fracture depends on energy considerations, as well as on strength considerations. Separation of an interface can only occur if the local stresses exceed the cohesive strength of the interface, and if there is sufficient work done by the applied loads (or sufficient energy released by the adherends) to create a new surface. Analyses that satisfy only one of the strength or energy criteria will result in lower bounds for the overall strength of the system. For example, the use of a local strength criterion alone leads to a lower-bound because the presence of any stress concentrations may cause the local stress to exceed the fracture stress at extremely low applied loads. One can envisage several alternative formulations based on various combinations of critical energy, stress or strain that would capture the essential aspects required for a fracture model. However, no matter how the details might be formulated, they will all exhibit features similar to a class of fracture model known as cohesive-zone models (CZM) (Needleman, 1987; Tvergaard and Hutchinson, 1992). These models can be naturally incorporated in numerical analyses, they combine both strength and energy criteria, and have been shown to provide an excellent tool for characterizing fracture in the presence of plastic deformation. Techniques for evaluating the strength and energy parameters for cohesive-zone models have been demonstrated for adhesively-bonded materials exhibiting large plastic deformations (Yang et al., 1999, 2000, 2001; Yang and Thouless, 2001). Furthermore, recent work by Cavalli et al. $(2004,2005)$ on resistance spot welds has demonstrated how the use of cohesive zones, at both the weld interface between the adherends and at the interface where pull-out occurs between the nugget and adherend, allows transitions between the two failure mechanisms (interface fracture and nugget pull-out) to be effectively described.

The work of Cavalli et al. (2005) showed how the cohesive-zone model could be used to predict the failure mechanisms and strengths of different geometries. However, the strength parameter for the cohesive zone had to be estimated from the constitutive properties of the aluminum, rather than being determined directly. It is this limitation that has been specifically addressed in the present study, in which a similar cohesive-zone approach was adopted to explore mode-I interfacial fracture of ultrasonic spot welds in aluminum. A combination of experimental and numerical analyses was used to determine both the characteristic strength and the fracture toughness of the interface in ultrasonic welds made with a specific range of energy input. It was then shown that these values could be used to predict the behavior of similar welds formed in a different geometry of joint. An accompanying paper focuses on mixed-mode failure of spot welds by pull-out (Zhou et al., 2006).

\section{Finite-element modeling}

\subsection{ContinuUm PROPERTIES OF THE ADHEREND}

An aluminum alloy, AA6111-T4, was used as the base material of the adherend. The commercial finite-element code ABAQUS ${ }^{\circledR}$ was used to model the ultrasonically 
welded joints. The continuum properties of the adherends were incorporated directly as a point-by-point representation of a tensile stress-strain curve obtained for the alloy with the appropriate heat treatment. The aluminum alloy was assumed to be isotropic and to follow a von Mises yield criterion. The hardening effect in any region where the adherend was bent during the fabrication process was included in the analyses by conducting a separate finite-element calculation to determine the initial plastic strain field.

Vickers hardness tests using micro-indentation were performed to investigate whether there were any local changes in hardness caused by the welding process itself. A typical ultrasonic weld between two $0.9 \mathrm{~mm}$ AA6111-T4 sheet metal coupons was cut through the center. The polished cross section of the weld was indented by a Vickers indenter with a diagonal of $15 \mu \mathrm{m}$. A series of indentations (each at $0.98 \mathrm{~N}$ for $10 \mathrm{~s}$ ) were made, starting from the base metal and traversing into the weld zone. This experiment showed that there was no significant change in hardness despite obvious evidence of extensive local plastic deformation and microstructural changes in the weld zone (Figure 1). In light of this result, the numerical results that follow assume that the material properties are uniform throughout the adherends.

\subsection{Cohesive zONE}

There are three key components of implementing a cohesive-zone model in finite-element analyses: (i) the use of user-defined elements to define a crack path (or paths); (ii) a traction-separation law integrated into these user-defined elements that provides an intrinsic relation between the tractions across the crack surface and the separation of the crack surfaces; (iii) a failure criterion that defines when the cohesive elements can no longer sustain a load, and, hence, when the crack advances. In the present work, the weld was represented by a region of 3-D user-defined cohesive-zone elements. These elements were placed over the entire interface between the adherends where experimental observations indicated that a welded interface had been formed. It should be appreciated that no process modeling was done in this study. The weld area was not calculated in any fashion; it was measured optically for each specimen after fracture. This measured area was a direct input to the numerical analysis of the joint.

Figure 2(a) shows a schematic illustration of the cohesive-zone element used in this study. Additional details are provided by Cavalli (2003) and Yang (2000). The two surfaces represented by the shaded areas are formed by two groups of nodes each belonging to a solid continuum element of adherend on either side of the crack.

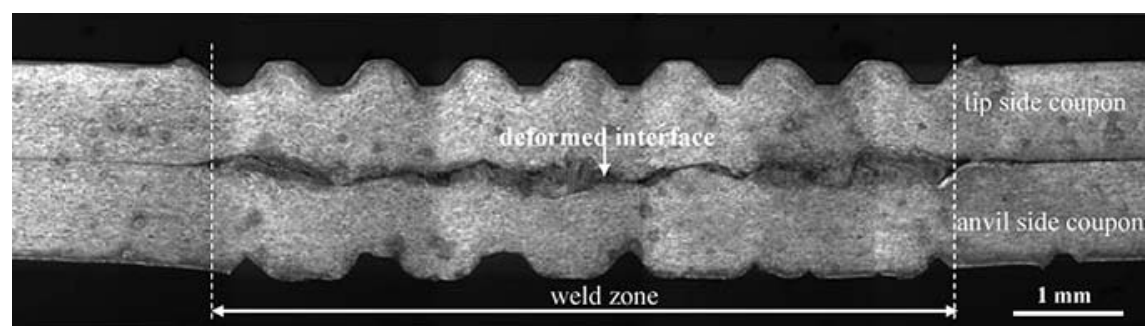

Figure 1. Optical micrograph of the weld region. 
(a)

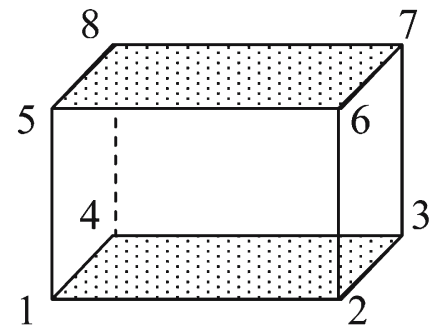

(b)

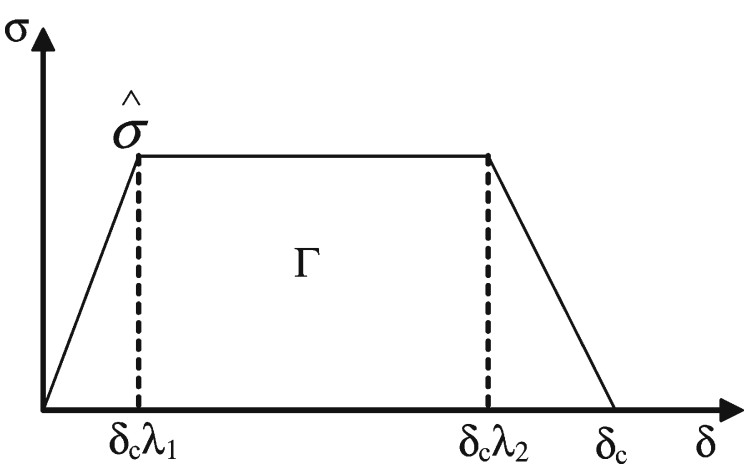

Figure 2. (a) A 3-D cohesive-zone element, and (b) the associated trapezoidal traction-separation law.

Relative displacements between node pairs occur independently, and cause deformation of the element. The local normal direction of the cohesive-zone element is perpendicular to a middle surface between the two shaded element faces, along which the opening mode of fracture (mode I) is defined. This direction and two orthogonal shearing directions (modes II and III) define a local Cartesian coordinate system of the element. Although the cohesive-zone element is fully capable of handling 3-D deformation, the geometries studied in the present work only exhibit mode-I deformation. The relative displacement, $\delta$, within a node pair is linked to the cracksurface traction, $\sigma$, through a trapezoidal shaped traction-separation law shown in Figure 2(b). This law is characterized by a characteristic strength, $\hat{\sigma}$, and mode-I toughness, $\Gamma_{I}$. The precise shape of the traction-separation curve does not generally affect the fracture behavior significantly (Tvergaard and Hutchinson, 1992; Li et al., 2005). In the present work, the trapezoidal shape was chosen to mimic approximately elastic-plastic behavior, and the two shape parameters, $\lambda_{1}$ and $\lambda_{2}$, were selected to avoid any significant numerical instabilities during the calculation $\left(\lambda_{1}=0.01\right.$ and $\lambda_{2}=$ $0.5)$. Failure of a node pair in mode-I fracture is defined as occurring when the separation reaches a critical value, $\delta_{c}$, or, equivalently, when the energy-release rate $\mathcal{G}_{\mathrm{I}}$ (defined as $\mathcal{G}_{I}=\int_{0}^{\delta} \sigma \mathrm{d} \delta$ ) equals the toughness, $\Gamma_{I}$. At this stage, the node pair no longer supports a load, and the crack advances.

In the finite-element modeling presented in this paper, the weld nugget was embedded in an adherend modeled by a refined mesh of continuum elements, while the user-defined elements covered an appropriate area representing the weld interface (Figure 3). Mesh-sensitivity analyses were conducted to ensure that the size of both the continuum elements and the cohesive-zone elements were fine enough to ensure convergent solutions within the desired level of uncertainty, which recognized the 
(a)

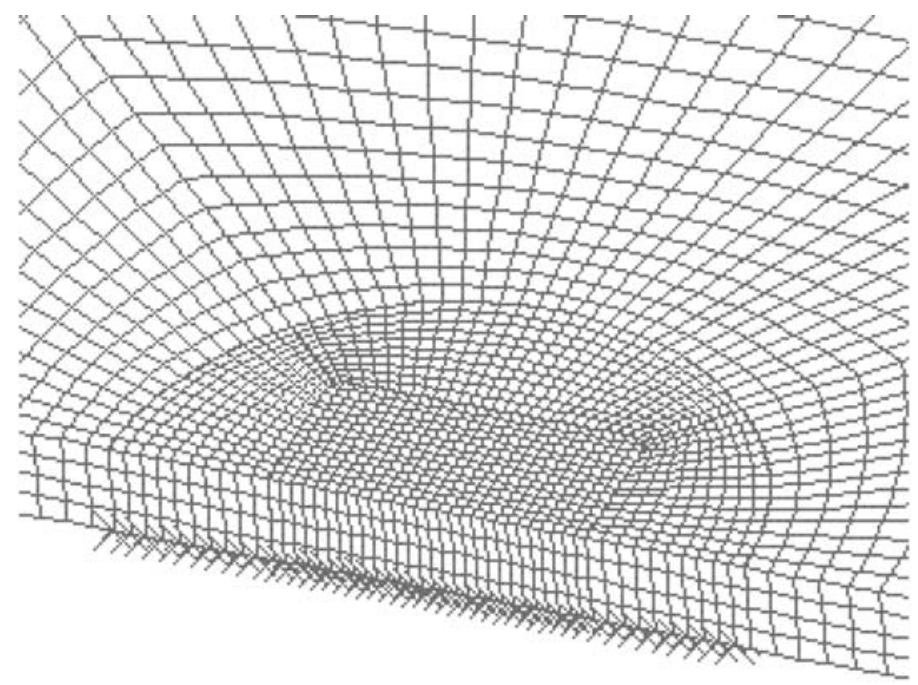

(b)

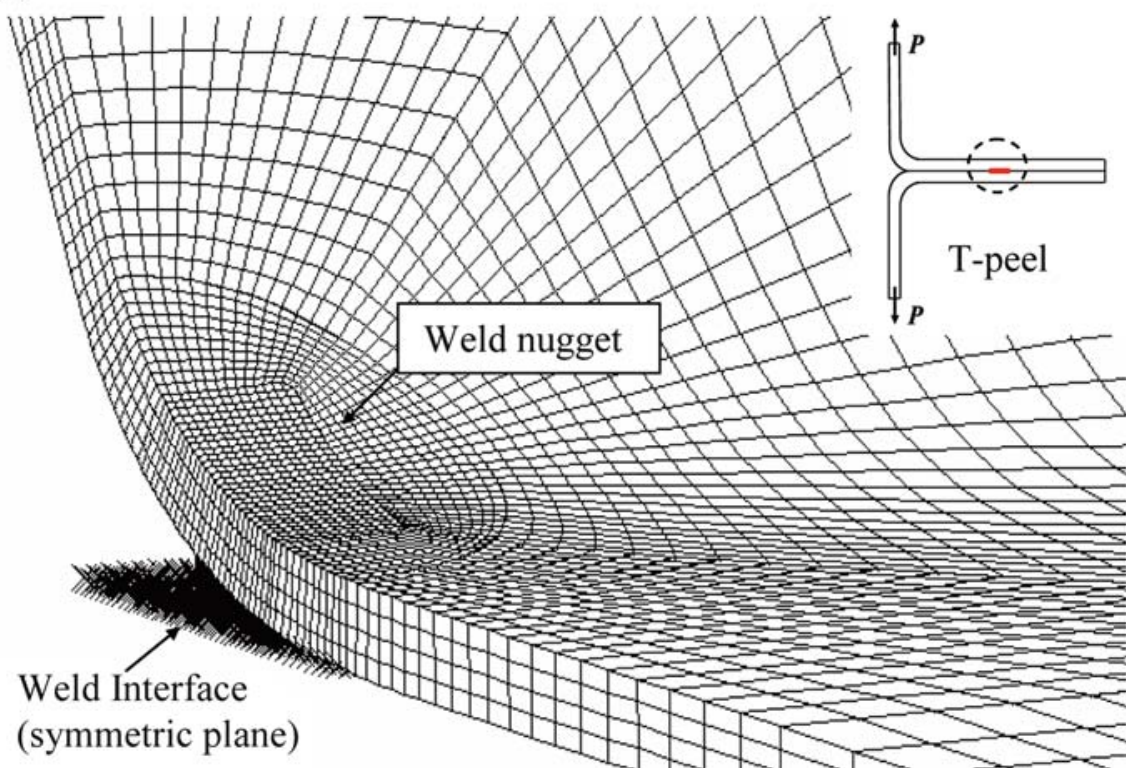

Figure 3. Finite-element simulation of mode-I interfacial fracture in a T-peel welded joint. (a) The original undeformed mesh and (b) the deformed mesh.

level of the underlying experimental uncertainty and variability. The typical finiteelement meshes used in the study had about 11,000 brick elements and 15,000 nodes. Attention in this initial paper was limited to situations for which weld pull-out did not occur, so that only nugget interface fracture was considered. It should be noted that the area of the weld was a crucial parameter in these analyses, because the failure mechanism and strength depend not only on the fracture parameters, but also on the size of the weld (Cavalli et al., 2005). The size of the weld, in turn, depends on the welding energy and the size of the welding tip (Harthoorn, 1978; Matsuoka, 1995). Throughout the present study, the welding parameters were held constant at 


\section{B. Zhou et al.}

a level that always ensured nugget fracture rather than pull-out. After the specimens failed at the interface, the nominal size of the area over which the weld had formed was measured optically. In the finite-element modeling, the area over which cohesive elements were located was defined by these post-fracture observations. It should be noted that in a typical industrial setting the welding parameters would be deliberately set to ensure that the weld area was large enough to ensure failure by pull-out. In the present paper, only cohesive failure was being studied. In a companion paper, the pull-out mechanism of failure is explored (Zhou et al., 2006).

\section{Sensitivity of load-displacement curves to fracture parameters}

It has been shown in previous studies that the mode-I cohesive parameters of adhesive joints can be determined by fitting experimental load-displacement curves to numerical predictions (Yang et al., 1999, 2000, 2001; Yang and Thouless, 2001; Li et al., 2005). However, adhesive joints typically have much greater bonded areas than spot-welded joints. This means that there is generally much more stable crack propagation associated with the failure of adhesive joints than there is with failure of spot-welded joints. As a result, the load-displacement curves obtained during fracture of a spot-welded joint are much less sensitive to the fracture parameters than are the curves obtained during failure of an adhesively-bonded joint. This makes it much more difficult to obtain values of the cohesive parameters for spot welds.

The problem, and a possible solution, is illustrated by a sensitivity study of the response of two geometries to variations in the interfacial fracture parameters. Figure 4 shows the details of a T-peel and a U-peel geometry used for this sensitivity study. Each geometry is assumed to consist of adherends made from an AA6111-T4 aluminum alloy, bonded with identical circular welds with a radius of $3.0 \mathrm{~mm}$; arbitrary values of $\Gamma_{I}=13 \mathrm{~kJ} \mathrm{~m}^{-2}$ and $\hat{\sigma}_{I}=290 \mathrm{MPa}$ were initially chosen for the mode-I fracture parameters of the weld. Figure 5 shows how the load varies as a function of the displacement between the loading points of the two geometries. Superimposed on these curves are essentially identical load-displacement plots that could be obtained using other combinations of the interfacial toughness and strength. For each geometry, multiple combinations of strength, $\hat{\sigma}_{I}$, and toughness, $\Gamma_{I}$, resulted in almost identical load-displacement curves. Therefore, one can conclude that it is not possible to obtain a unique pair of fracture parameters that fits a given load-displacement curve for a single joint geometry. However, it should be noted that, other than the original pair of fracture parameters chosen to represent the 'actual' properties of the weld, no other pair of parameters describes the behavior of both geometries. Figure 6 illustrates this point by showing plots of the pairs of fracture parameters that produce identical load-displacement curves. This figure suggests that it may be possible to determine a unique pair of fracture parameters using fits to experimental curves from two different geometries with identical welds. A pair of parameters that fits both geometries would be the correct set to represent the weld. Although this approach is conceptually appealing, experimental uncertainty might cause considerable difficulties in identifying the fracture parameters to a reasonable degree of certainty, especially when the sensitivities of the two geometries are as similar as those shown in Figure 6 . This approach is practical only if the two geometries have substantially different sensitivities to the toughness and the strength. 

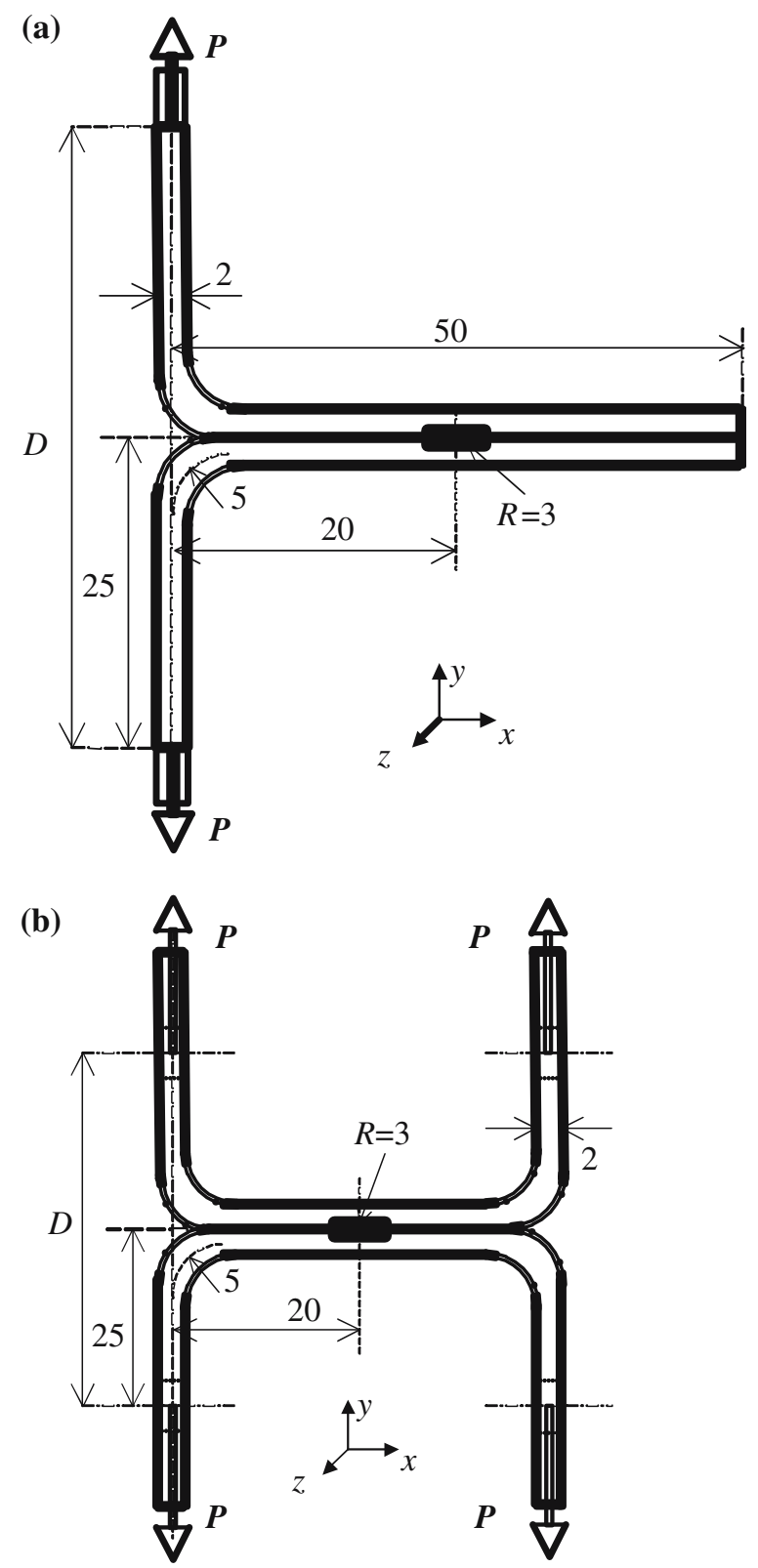

Figure 4. (a) T-peel and (b) U-peel geometries used in the sensitivity study. Dimensions in mm. The out-of-plane dimension is $5 \mathrm{~mm}$.

Fortunately, geometries similar to that shown in Figure 7, where a joint with a relatively small bonded region is attached to a rigid substrate and then pulled in direct tension, appear to have a strength that is reasonably independent of the toughness. Specifically, cohesive-zone analyses indicated that the maximum load that could be supported by such a geometry was essentially identical to the cohesive strength of the weld multiplied by the weld area. ${ }^{1}$ 


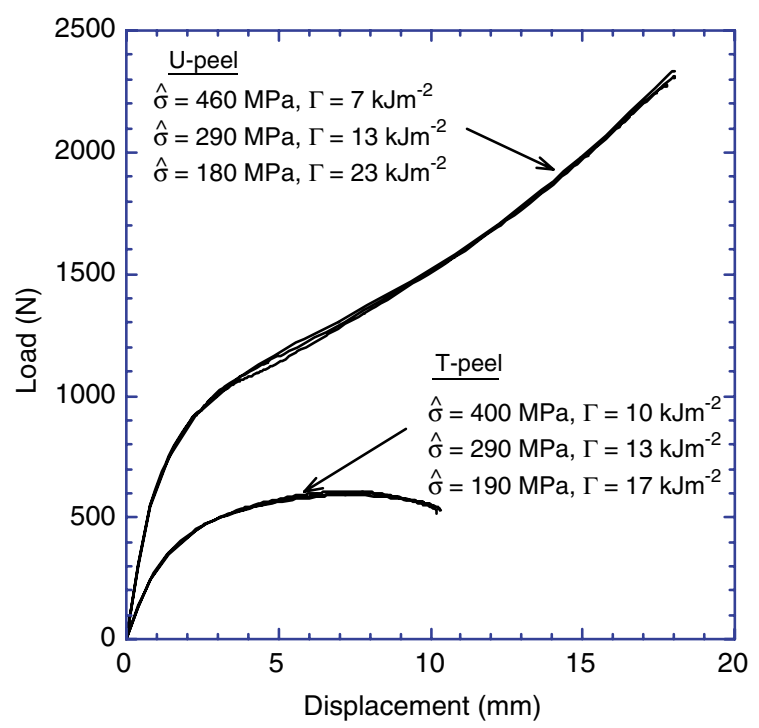

Figure 5. Multiple combinations of two fracture parameters result in almost identical loaddisplacement curves for the T-peel and U-peel geometries shown in Figure 4. The properties of an AA6111-T4 aluminum alloy were used to model the deformation of the adherends.

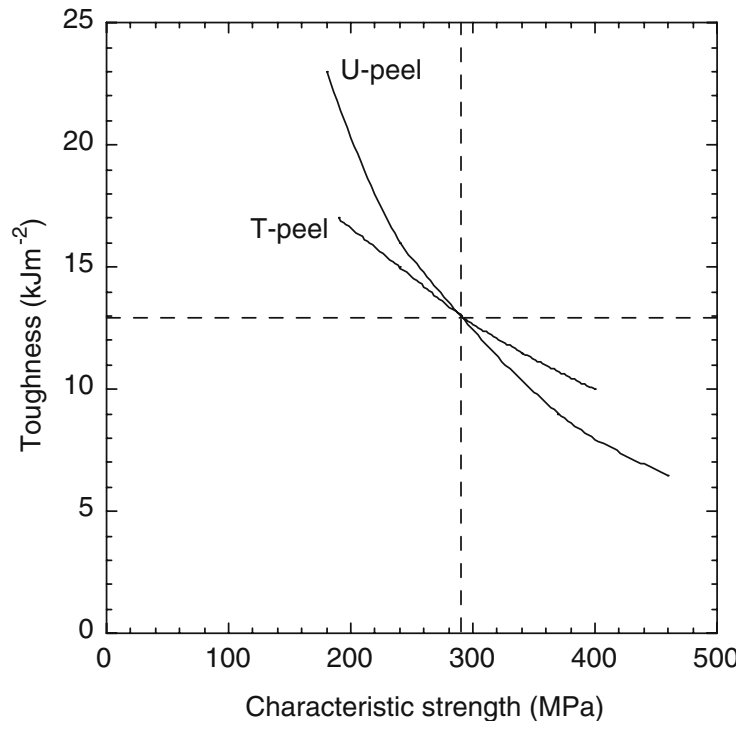

Figure 6. Correlation between pairs of fracture parameters that yield essentially identical similar loaddisplacement curves for the T-peel and U-peel geometry shown in Figure 4.

\section{Determining the interface strength}

To prepare the specimens shown in Figure 7, aluminum coupons were bonded using an ultrasonic welder ${ }^{2}$ with a rectangular tip and at the same welding energy used throughout this study. The coupons were then cut to form $50 \mathrm{~mm} \times 25 \mathrm{~mm}$ overlapping joints. Steel backing plates were adhesively bonded to the exposed surfaces of these coupons using a commercial structural adhesive. ${ }^{3}$ The finished specimens were 

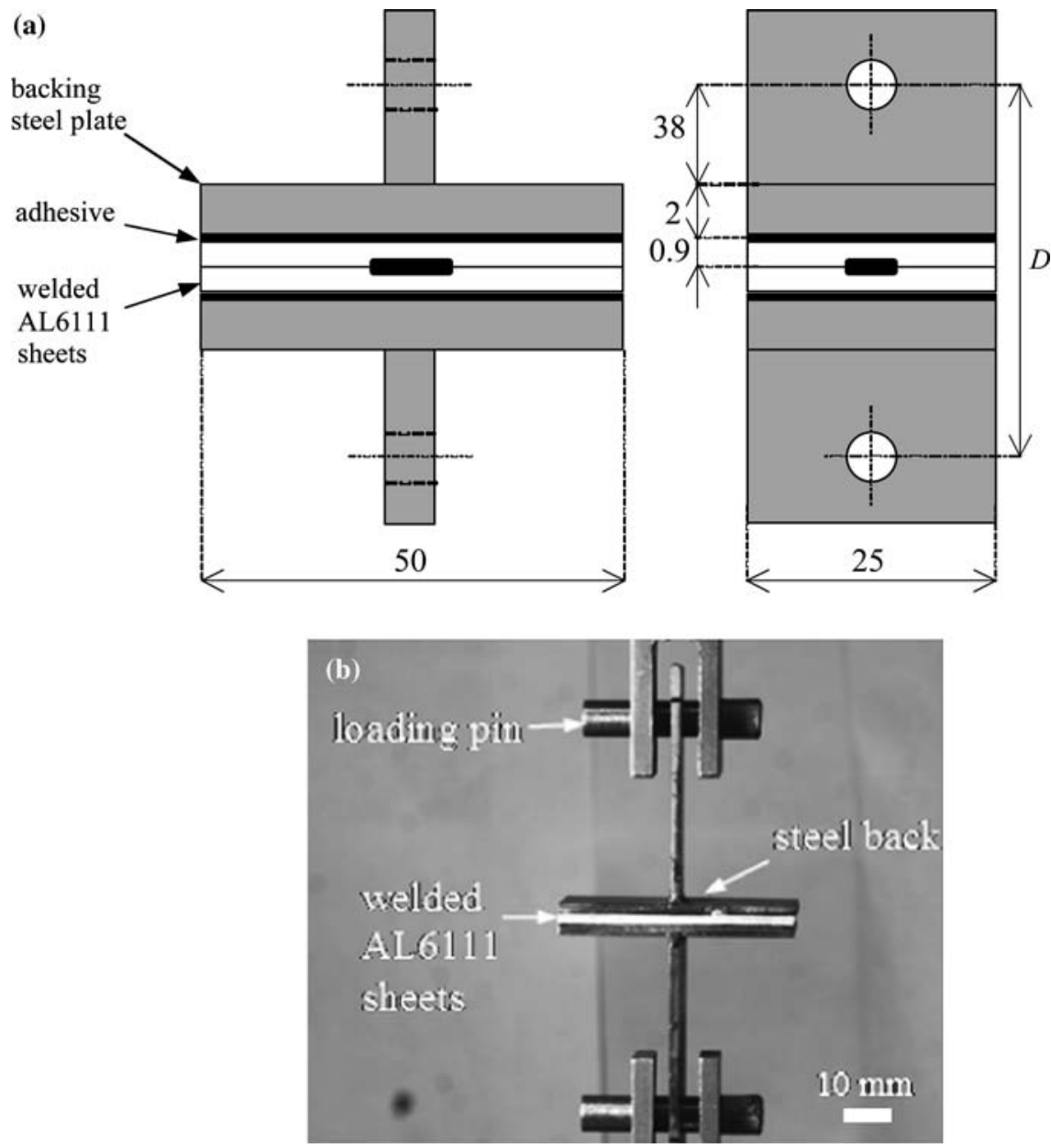

Figure 7. (a) Specimen used for a direct uniaxial tensile test used to measure the mode-I characteristic strength of weld interface. (b) Photograph of test in progress.

then placed in a furnace for $30 \mathrm{~min}$ to cure the adhesive at $180^{\circ} \mathrm{C}$. The specimens were tested on a screw-driven tensile test machine at a displacement loading rate of $0.2 \mathrm{~mm} / \mathrm{min}$. In all eleven tests done, the welds failed at the interface. There was no discernable plastic deformation or damage of the backing plate; nor was there any obvious damage to the adhesive or to the aluminum adherends.

After fracture, the nominal weld area for each specimen was measured using an optical microscope. In all cases, the nominal area of the weld was estimated as the rectangular area bordering the fractured surface, as shown by the dashed lines in the typical micrograph of a fractured weld shown in Figure 8. The average nominal strength of the welds, determined by dividing the peak load by the nominal weld area, was determined to be $83 \pm 6 \mathrm{MPa}$.

As can be seen from Figure 9, the nominal strength of the welds appears to be independent of the nominal area when a fixed weld energy is used. The independence of the nominal strength from the weld area indicates that the nominal strength can probably be identified with the cohesive strength of the weld. This was verified by performing cohesive-zone analyses of the geometry shown in Figure 7 with a 


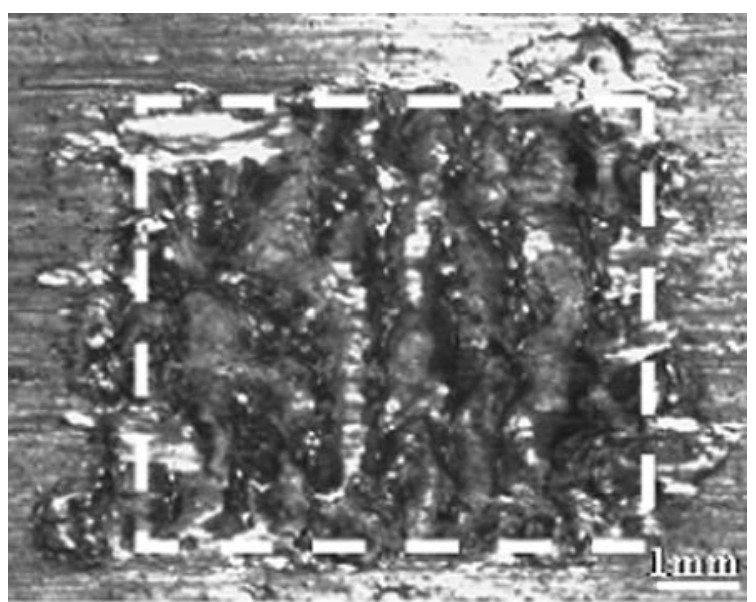

Figure 8. Micrograph of the interface of an ultrasonic weld fractured in mode I. The dotted white line shows the region that was identified as the nominal bond area.

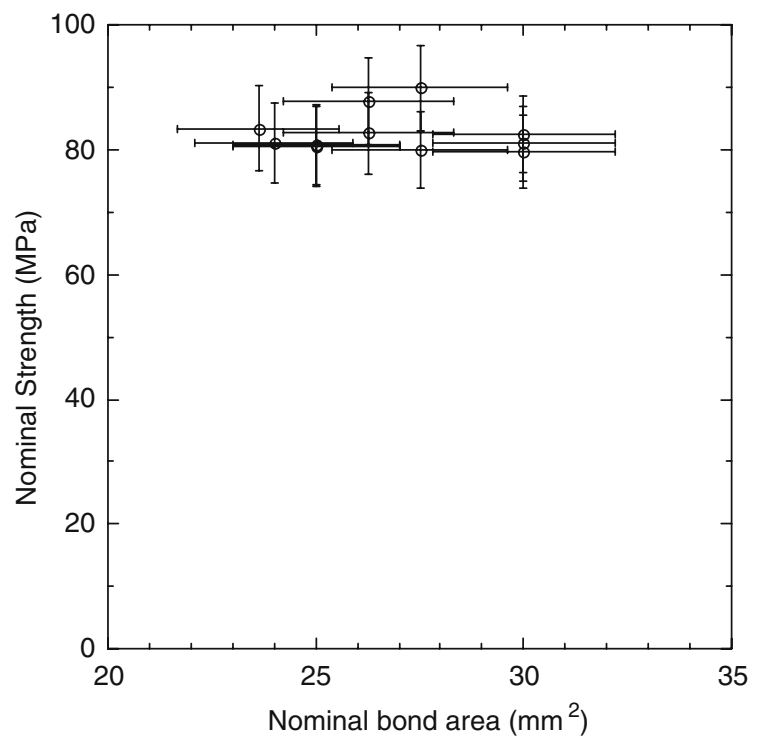

Figure 9. Average nominal strength of the ultrasonic welds plotted as a function of the nominal bond area.

rectangular bonded region of $27 \mathrm{~mm}^{2}$ (which corresponds to the average of the nominal weld areas observed in the tests). A trapezoidal traction-separation law of the form shown in Figure 2 was used, with a fixed cohesive strength of $83 \mathrm{MPa}$, but with different values of toughness. The load-displacement curves that resulted from this analysis are shown in Figure 10. These plots verify that the cohesive strength was indeed equal to the nominal strength of this configuration, and that the peak load is controlled by the cohesive strength, not by the toughness. This result was associated with the relatively uniform stress distribution across the weld which, in turn, was associated with a fracture-length scale $E^{\prime} \Gamma_{I} / \hat{\sigma}_{I}^{2}$ (where $E^{\prime}$ is the effective modulus of the system including the steel tabs) being greater than the size of the weld. It is this 


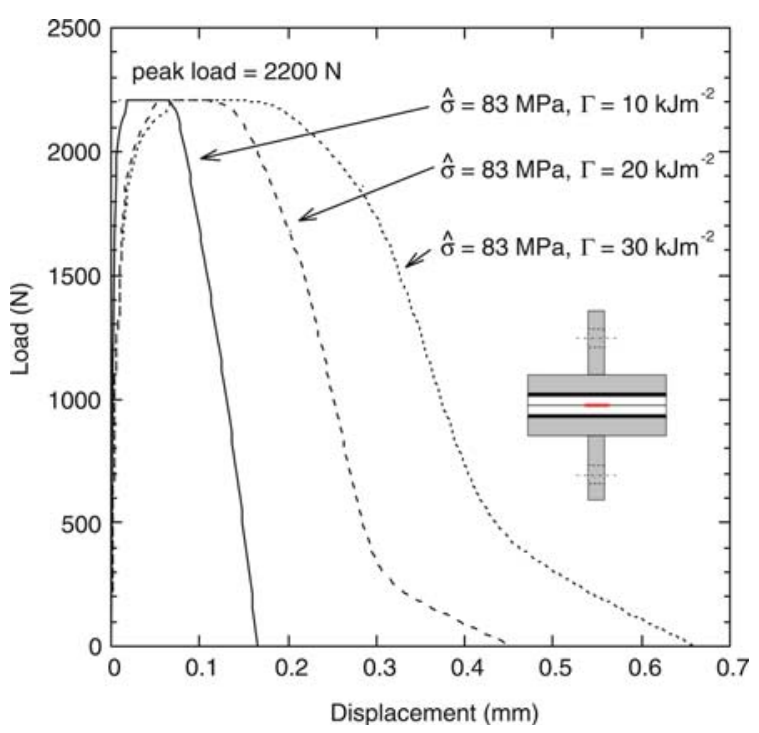

Figure 10. Finite-element simulations of the direct tensile test show that the peak load is uniquely determined by the characteristic strength of weld interface, regardless of the interface toughness. An average bond area of $A=27 \mathrm{~mm}^{2}$ was used in these calculations.

characteristic that made the geometry so useful for determining the cohesive strength directly.

These results, and additional cohesive-zone analyses, confirmed that the cohesive strength of the ultrasonic welds formed in this study was $83 \pm 6 \mathrm{MPa}$. It is interesting to note that this value is much lower than the tensile strength of the aluminum alloy, indicating that the bonding across the interface of these welds was not complete at the levels of welding energy used in this study. ${ }^{4}$

\section{Determining the interfacial toughness}

Once the mode-I cohesive strength of the weld has been determined, the toughness can be found from tests using a different geometry that has sensitivity to the toughness. Figure 6 suggests that a T-peel test is a suitable geometry to determine the toughness of spot welds, once the cohesive strength is known.

A series of ten T-peel test specimens were prepared by using a single ultrasonic weld to bond two $0.9 \mathrm{~mm}$ thick AA6111-T4 aluminum coupons together. The same level of welding energy was used to make this weld as had been used to make the direct-tension specimens. The nominal weld areas for these joints were in the range of $20 \pm 2 \mathrm{~mm}^{2}$. The coupons were $25 \mathrm{~mm}$ wide, and $125 \mathrm{~mm}$ long, with the center of the weld being $30 \mathrm{~mm}$ from one end. The welded coupons then went through the same heat treatment used in the direct tensile tests, and were bent into the shape shown in Figure 11(a). A prescribed loading displacement of $5 \mathrm{~mm} / \mathrm{min}$ was applied to the specimen by clamping it with wedge grips on a screw-driven machine. A CCD camera took a series of images of the tests, so that the cross-head displacement could be calibrated. Figure 11(b) shows the deformation of a typical specimen close to final failure. The small rotation that can be observed in this figure indicates a small asymmetry in the geometry or loading that was not modeled numerically. 

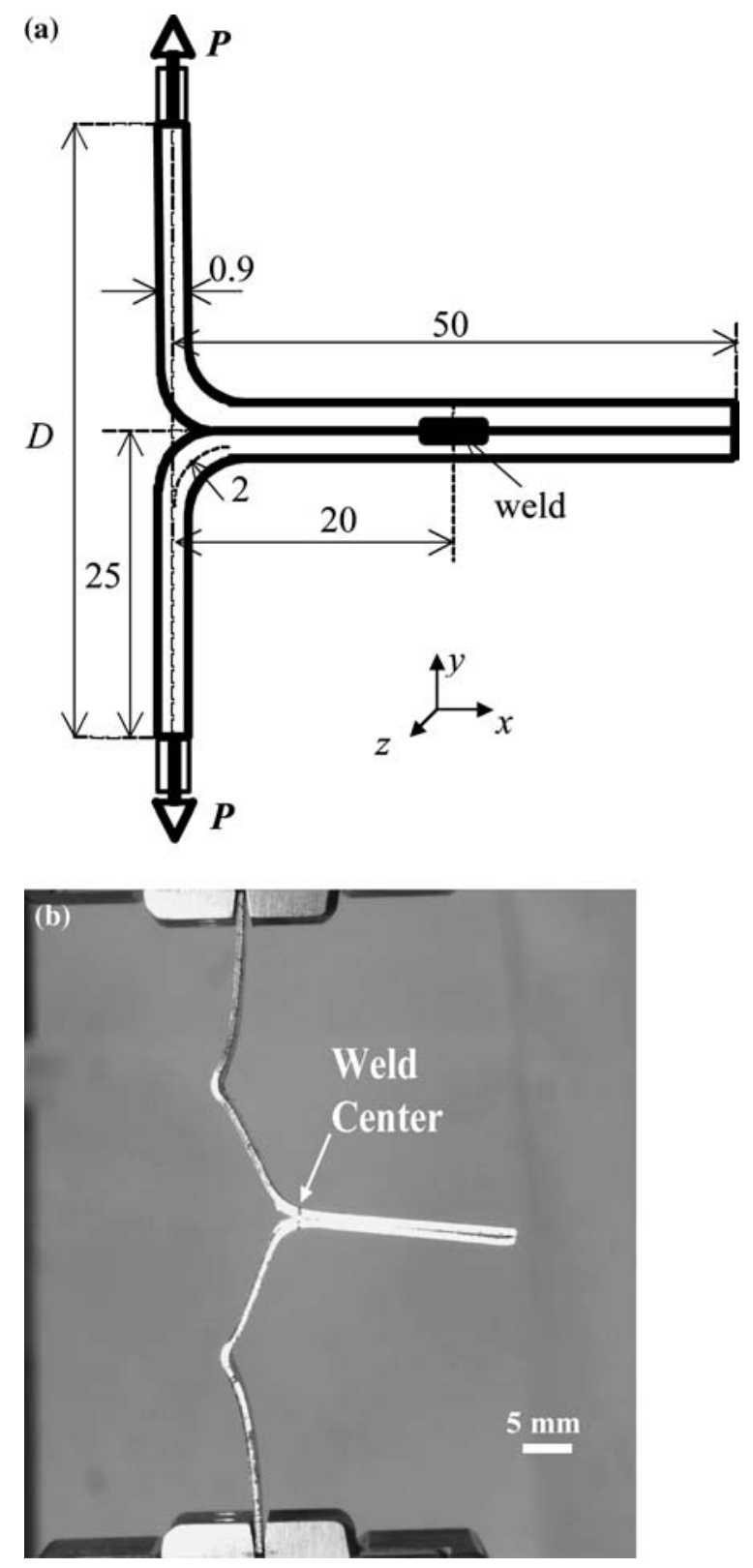

Figure 11. (a) Configuration and dimensions (in $\mathrm{mm}$ ) of the T-peel joints used to determine the toughness. (The out-of-plane dimension is $5 \mathrm{~mm}$ ). (b) A photograph of the loaded T-peel specimen just before fracture.

The load-displacement plots that were obtained from these experiments are shown in Figure 12.

The geometry was analyzed using cohesive-zone analyses with a traction-separation law of the form shown in Figure 2(b). The constitutive properties of the baked aluminum alloy were used in the analysis to model the deformation of the adherends. These constituive properties were input into the numerical model as a point-by-point represenation of a tensile test. The salient parameters that described the alloy are summarized 
Table 1. Summary of the approximate mechanical properties of the aluminum alloy, as represented in the numerical analyses.

\begin{tabular}{ll}
\hline AA6111-T4 & Baked for $30 \mathrm{~min}$ at $180^{\circ} \mathrm{C}$ \\
\hline Young's modulus & $69 \mathrm{GPa}$ \\
Poisson's Ratio & 0.3 \\
Tensile proportional limit & $159 \mathrm{MPa}$ \\
Ultimate tensile strength & $397 \mathrm{MPa}$ \\
\hline
\end{tabular}

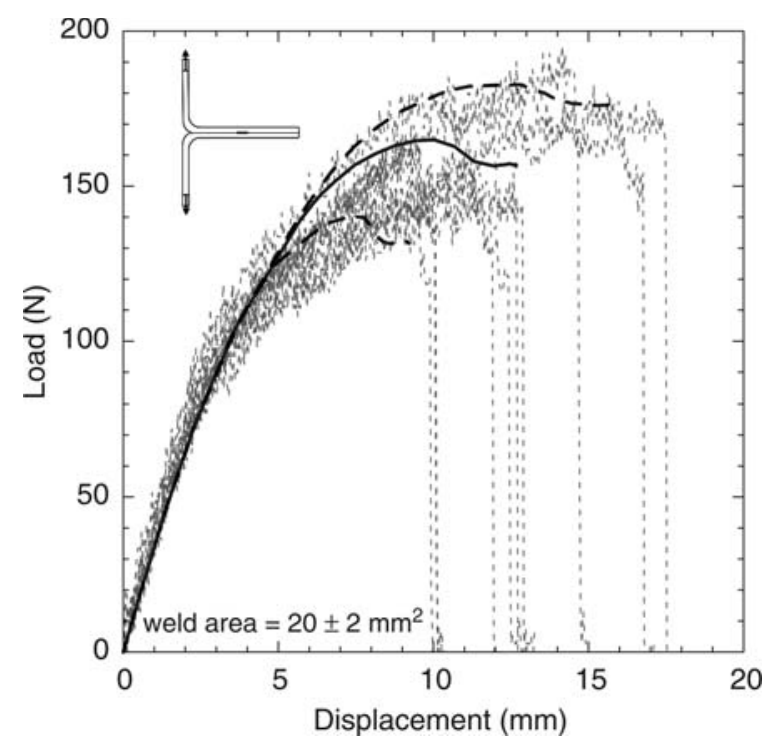

Figure 12. Comparison between the numerical fits (with upper and lower bounds) and the experimental load-displacement curves for T-peel tests. This comparison indicated that the mode-I toughness of the interface was $11 \pm 3 \mathrm{kJm}^{-2}$.

in Table 1. The cohesive strength for the traction-separtaion law was varied between 77 and $89 \mathrm{MPa}(83 \pm 6 \mathrm{MPa})$, and different values of toughness were used to compute numerical load-displacement curves for the geometry. Comparisons were made between the experimental curves shown in Figure 12, and these different predictions. The curves that provided the best fits to the experimental data were assumed to give the appropriate values of toughness. These best-fit curves are superimposed on the experimental data of Figure 12, and correspond to a toughness of $11 \pm 3 \mathrm{kJm}^{-2}$.

It should be noted that the stress distribution in the weld is not uniform in the T-peel geometry. Therefore, the nominal strength of a T-peel joint does not represent the cohesive strength of the weld; it is affected by the toughness as well as by the strength. In particular, it will be observed that the nominal strength of the ultrasonic welds (maximum load divided by nominal weld area) in the T-peel geometry was in the range of 7-11 MPa, essentially an order of magnitude lower than the cohesive strength. This illustrates the importance of including an energy term as the second fracture criterion for spot welds; a strength criterion alone cannot give a predictive indication of the performance of a weld when the joint geometry is changed significantly. 

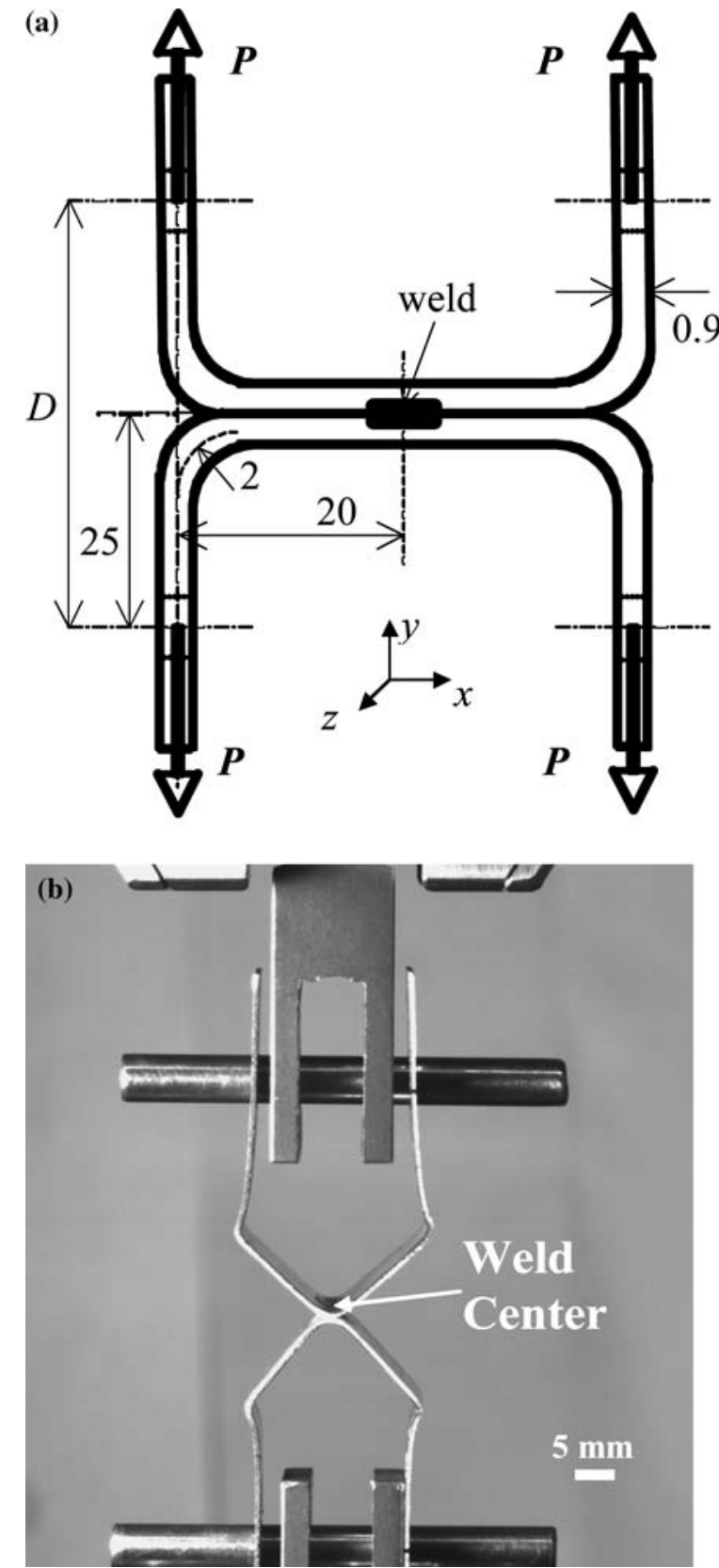

Figure 13. (a) Configuration and dimensions (in $\mathrm{mm}$ ) of the U-peel joints used to verify the values of the cohesive parameters. (The out-of-plane dimension is $5 \mathrm{~mm}$ ). (b) A photograph of the loaded U-peel specimen just before fracture.

\section{Predictive use of the fracture parameters}

U-peel specimens (Figure 13a), were then used as an independent third geometry to verify that the two mode-I fracture parameters could be used in a predictive fashion. These specimens had the same heat treatment as the previous specimens, and were welded using the same equipment and welding parameters. Post-fracture examination 
of the welds indicated that the weld areas in these specimens were $19 \pm 2 \mathrm{~mm}^{2}$. The specimens were pin-loaded by a screw-driven test machine, and were tested at a displacement rate of $5 \mathrm{~mm} / \mathrm{min}$. Figure 13(b) shows a photograph of a typical loaded joint just before fracture.

For the numerical analyses, $1 / 8$ of the geometry was modeled using the measured areas of the welds, the constitutive properties of the baked aluminum, and the mode-I fracture properties of the weld determined from the previous two geometries. Figure 14 compares numerical predictions for the load-displacement curves that were obtained using these parameters to the experimentally obtained curves. It is clear that the cohesive-zone model provides an excellent prediction of the performance (strength and energy absorption) of the new geometry. It is also interesting to note that a change in the geometry of the joint from a T-peel to a U-peel configuration results in an increase in the strength by a factor of about three. This increase results from the slightly more uniform stress distribution, associated with the symmetry of the Upeel geometry. However, even with this configuration, the bending of the arms does cause a non-uniform stress distribution within the weld, resulting in a nominal weld strength that is still substantially less than the cohesive strength of the weld. The changes in strength with geometry were successfully predicted by the cohesive-zone simulations. These results are further evidence that a strength parameter alone is not sufficient to describe the fracture behavior of a spot weld. Only with the correct combination of strength and energy parameters, can fracture of spot welds in different joint configurations be successfully predicted.

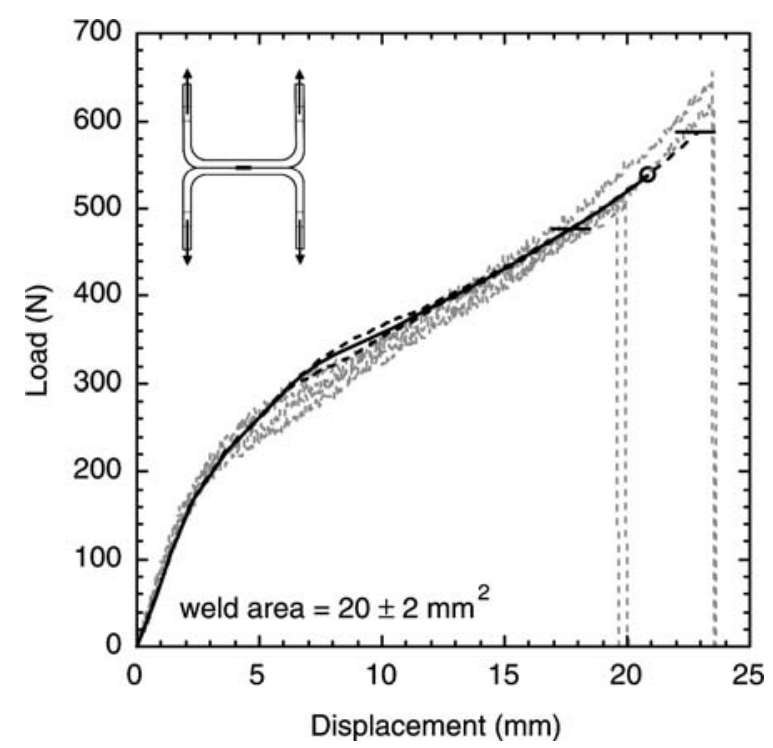

Figure 14. Comparison between the numerical predictions (with upper and lower bounds) and the experimental load-displacement curves for U-peel tests. The fracture parameters used in the numerical analysis were $\hat{\sigma}_{I}=83 \pm 6 \mathrm{MPa}$ and $\Gamma_{I}=11 \pm 3 \mathrm{~kJ} / \mathrm{m}^{-2}$ The predicted peak loads are indicated by a small circle for the mean values of the cohesive parameters, and by horizontal bars for the lower and upper bounds of the cohesive parameters. 


\section{B. Zhou et al.}

\section{Concluding remarks}

Ultrasonic welds have been used as a model system to investigate how the mode-I cohesive parameters of a spot weld may be determined using cohesive-zone finiteelement analyses and fracture tests. It was found that load-displacement curves from a single geometry cannot be used to determine a unique pair of the strength and toughness parameters. However, in the particular system studied here, uniaxial tensile tests did allow the cohesive strength to be measured directly as the nominal strength of the welds. Subsequent tests with a T-peel geometry then allowed the toughness to be determined by comparing experimental load-displacement curves to numerical predictions obtained from cohesive-zone analyses. For the particular welds studied in this paper, the cohesive strength was determined to be $\hat{\sigma}_{I}=83 \pm 6 \mathrm{MPa}$, and the interface fracture toughness was determined to be $\Gamma_{I}=11 \pm 3 \mathrm{kJm}^{-2}$. These two parameters were then used to predict the performance of a third configuration. The changes in strength and energy absorption associated with changes in geometry were successfully captured by the cohesive-zone model. Future work will develop models for mixed-mode fracture of the interface, and for nugget pull-out. It is expected that these will allow a full understanding of the parameters required to predict the strength, and the transitions between interface failure and nugget pull-out in spot welds.

This work has demonstrated that cohesive-zone analyses with properly calibrated fracture parameters may provide an excellent predictive tool to describe interface failure of spot welds. However, the high quality of the predictive nature of the technique comes at a high computational cost, compared with some of the more traditional approaches which may be less versatile in their predictive capabilities. Clearly, practical implementations of cohesive-zone approaches for modeling weld failure in largescale structures must involve investigations of how to balance performance against computational cost. It is expected that as computational costs continues to decrease, there will be an increased motivation to focus on these more predictive approaches for modeling fracture.

\section{Acknowledgements}

The support through NIST ATP Cooperative Agreement 70NANB3H3015 on ultrasonic metal spot welding is gratefully acknowledged. The authors also thank D.J. Hill, R. Jahn, L. Reatherford, D. Wilkosz, E. Hetrick, A. Grima, and R. Cooper at Ford SRL for their help in this work.

\section{Notes}

1 As a general comment, it should be noted that a distinction has to be made between the cohesive strength of an interface (meaning the maximum stress the interface can support) and the characteristic strength of an interface (related to the average strength and associated with the dominant toughening mechanism). In geometries with relatively large bonded regions, it tends to be the characteristic strength, along with the toughness, that governs fracture. In geometries with very small geometrical features, the cohesive strength can become important ( $\mathrm{Li}$ et al., 2005). However, in the trapezoidal traction-separation law of Figure 2 chosen to model the interface in this study, there is very little distinction between these two quantities. 
2 Sonobond Ultrasonics WeldMaster ${ }^{\mathrm{TM}}$ MH-2014D.

3 Dow Betamate ${ }^{\circledR} 4601$ structural adhesive.

4 Ultrasound images of the welds show that only partial bonding may have occurred across the interface at the energy levels used in this study (Ghaffari et al., 2005). Additional studies conducted as part of this work indicated that the nominal strength of the interface increased when more energy was used to form an ultrasonic spot weld. This suggests that the level of bonding may increase with weld energy, as well as the nominal bond area. However, a parametric study to look at this effect was not the intent of this work.

\section{References}

Cavalli, M.N. (2003). Cohesive-zone modeling of structural joint failure, Spot-Welded Joints. Ph.D. Dissertation, University of Michigan, Ann Arbor, MI.

Cavalli, M.N., Thouless, M.D. and Yang, Q.D. (2004). Cohesive-zone modeling of the deformation and fracture of weld-bonded joints. Welding Journal. 83, 133S-139S.

Cavalli, M.N., Thouless, M.D. and Yang, Q.D. (2005). Cohesive-zone modeling of the deformation and fracture of spot-welded joints. Fatigue and Fracture of Engineering Materials and Structures 28, 861-874.

Chang, B.H., Shi, Y.W. and Dong, S.J. (1999). Comparative studies on stresses in weldbonded, spotwelded and adhesive bonded joints. Journal of Materials Processing Technology 87, 230-236.

Deng, X., Chen, W. and Shi, G. (2000). Three-dimensional finite element analysis of the mechanical behavior of spot-welds. Finite Elements in Analysis and Design 35, 17-39.

Ghaffari, B., Hetrick, E.T., Mozurkewich, G. and Reatherford, L.V. (2005). In: Review of Progress in Quantitative Nondestructive Evaluation (edited by Thompson, D.O. and Chimenti, D.E.), Vol. 24B. Am. Inst. Phys., 1197-1203.

Harthoorn, J.L. (1978). Ultrasonic metal welding. Ph.D. Dissertation, Technical College Eindhoven, Netherlands.

Hazlett, T.H. and Ambekar, S.M. (1970). Additional studies on interface temperature and bonding mechanisms of ultrasonic welds. Welding Journal 49, 196-200.

Hetrick, E., Jahn, R., Reatherford, L., Skogsmo, J., Ward, S.M., Wilkosz, D., Devine, J., Graff, K. and Gehrin, R. (2005). Ultrasonic spot welding: a new tool for aluminum joining. Welding Journal 84, 6-30.

Jones, J.B. and Meyer, F.R. (1958). Ultrasonic welding of structural aluminum alloys. Welding Journal 3, 81-92.

Lee, Y.L., Wehner, T.J., Lu, M.W., Morrissett, T.W. and Pakalnins, E. (1998). Ultimate strength of resistance spot-welds subjected to combined tension and shear. Journal of Testing and Evaluation 26, 213-219.

Li, S, Thouless, M.D., Waas, A.M., Schroeder, J.A. and Zavattieri, P.D. (2005). Use of mode-I cohesivezone models to describe the fracture of an adhesively-bonded polymer-matrix composite. Composite Science \& Technologies 65, 281-293.

Lin, S.H., Pan, J., Wu, S.R., Tyan, T. and Wung, P. (2001). Failure loads of spot-welds under combined opening and shear static loading conditions. International Journal of Solids and Structures 39, 19-39.

Matsuoka, S. (1995). Ultrasonic welding and characteristics of glass-fiber reinforced plastic: comparison between the paper-making method and the impregnation method. Journal of Materials Processing Technology 55, 427-431.

Needleman, A. (1987). A continuum model for void nucleation by inclusion debonding. Journal of Applied Mechanics 54, 525-531.

Satoh, T., Abe, H., Nishikawa, K. and Morita, M. (1991). On three-dimensional elastic-plastic stress analysis of spot-welded joint under tensile shear load. Transactions of the Japan Welding Society 22, 46-51.

Tsujino, J., Hidai, K., Hasegawa, A., Kanai, R., Matsuura, H., Matsushima, K. and Ueoka, T. (2002). Ultrasonic butt welding of aluminum alloy and stainless steel plate specimens. Ultrasonics 40, 371-374. 


\section{B. Zhou et al.}

Tvergaard, V. and Hutchinson, J.W. (1992). The relation between crack growth resistance and fracture process parameters in elastic-plastic solids. Journal of the Mechanics and Physics of Solids 40, 1377-1397.

Wung, P. (2001). A force-based failure criterion for spot-weld design. Experimental Mechanics 41, 107-113.

Wung, P., Walsh, T., Ourchane, A., Stewart, W. and Jie, M. (2001). Failure of spot welds under in-plane static loading. Experimental Mechanics 41, 100-106.

Yang, Q.D., Thouless, M.D. and Ward, S.M. (1999). Numerical simulation of adhesively bonded beams failing with extensive plastic deformation. Journal of the Mechanics and Physics of Solids 47, 1337-1353.

Yang, Q.D., Thouless, M.D. and Ward, S.M. (2000). Analysis of the symmetrical $90^{\circ}$-Peel test with extensive plastic deformation. Journal of Adhesion 72, 115-13.

Yang, Q.D., Thouless, M.D. and Ward, S.M. (2001). Elastic-plastic mode-II fracture of adhesive joints. International Journal of Solids and Structures 38, 3251-3262.

Yang, Q.D. and Thouless, M.D. (2001). Mixed-mode fracture analysis of plastically deforming adhesive joints. International Journal of Fracture 110, 175-187.

Yang, Q.D. (2000). Fracture analyses of plastically-deforming adhesive joints. Ph.D. Dissertation, University of Michigan, Ann Arbor, MI.

Zhou, B., Thouless, M.D. and Ward, S.M. (2006). Predicting failure by nugget pull-out of ultrasonic spot welds in aluminum sheet metal. (submitted). 